\title{
Software review: Communication optimisation - The new mantra of database marketing. Fad or fact?
}

\author{
Received: 15th October, 2001
}

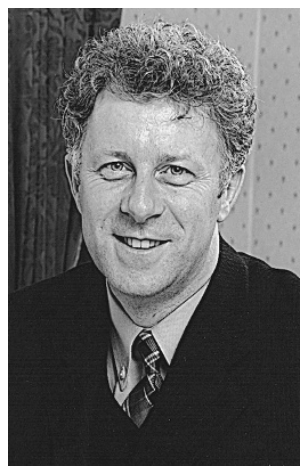

\section{Shaun Doyle}

is VP of Intelligent Marketing Solutions at SAS. He is responsible for driving the application of SAS technologies in marketing. Prior to this role he was Chairman of Intrinsic, a campaign management software company. A co-founder of the company with Steve Treadwell, he was responsible for management of the business consultancy team and the strategic direction of the company. He has extensive experience in the design, development, implementation and exploitation of customer-focused database marketing systems in a number of business sectors. These include financial services, retail, leisure, charity and telecommunications. He has been involved in the design and building of over 50 marketing databases in blue chip organisations.

$\mathrm{He}$ is well known in the industry, regularly speaking and running specialist workshops at leading industry conferences. He has had a number of papers published in the UK and overseas on the application of database marketing.

Abstract This paper describes the key components of analytical customer relationship management (CRM) and then explores the introduction of optimisation technology as a key component of an analytical CRM solution.

\section{INTRODUCTION}

It is interesting to see that like the youth of today, an industry such as marketing can be just as big a fashion victim. In the past it was campaign management, then marketing automation and now customer relationship optimisation. The term, originally used by NCR as a product name for one of their customer relationship management (CRM) solutions has now become the new mantra of the database marketing industry. It even has its own Gartner quadrant and associated acronym, 'CRO'. The same vendors appear as before, it is

\section{Shaun Doyle}

SAS Software Ltd, Units 63-64, Shrivenham One Hundred Business Park, Shrivenham, Swindon, Wilts SL7 2EB.

Tel: +44 (0)1367 240003; Fax: +44 (0)1367 244604; e-mail: shaun.doyle@ suk.sas.com just the positioning that appears to have changed. No wonder many on the client side are confused by the marketing hype.

This paper hopes to blow the froth off the hype and see if there is some real advance in optimisation technology that can make a material difference to the effectiveness of database marketing.

\section{ANALYTICAL CRM}

There is no doubt that analytical CRM solutions can help organisations intelligently manage the customer communication process more effectively. This benefit is augmented when deployed across multiple channels. In many cases this increased communication effectiveness is achieved while balancing channel capacity and other business constraints/objectives. This balanced approach ensures the channel resource is focused on the most valuable interactions.

It is only through this intelligent and balanced use of customer communications that an organisation can enhance the value of customer relationships while optimally exploiting the use of valuable resources.

These new analytical CRM solutions use optimisation technologies to allow marketers to deliver the most effective 


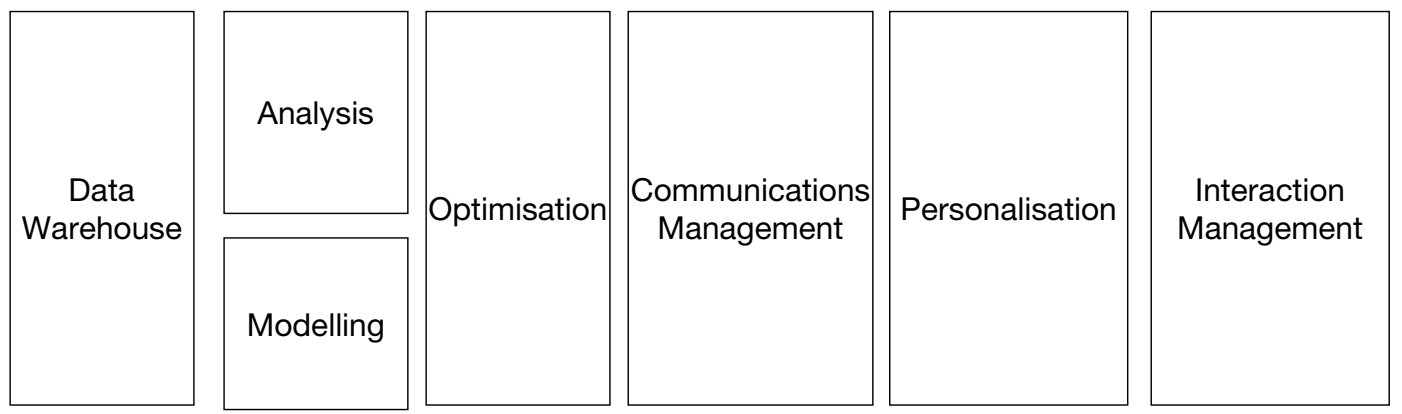

Figure 1 Components of analytical CRM

mix of messages and offers to each customer based on priority and the availability of resources within a particular time period.

In some cases this communication decisioning process is happening in real time.

\section{ANALYTICAL CRM SOLUTION FRAMEWORK}

The following section explores how optimisation technologies fit within the analytical CRM solution framework.

There are typically six areas of functionality found in comprehensive analytical CRM solutions. These are:

— analysis

— modelling

- communication

- personalisation

- optimisation

— interaction management.

These areas of functionality allow an organisation to analyse, model and predict customer behaviour while planning and automating personalised communications with individual customers across all channels. The following sections look at these functional components in more detail.

\section{ANALYSIS}

In order to manage customer communications more effectively it is essential that an organisation can analyse the characteristics and behaviour of its customers. This analytical capability needs to be embedded into the campaign management process if intelligence is to clearly drive these and other activities. This analytical capability is concerned with allowing an organisation to:

— understand the profile of customer groups

- compare the profile of different groups (respondent-non-respondent comparison)

- understand customer behaviour

- analyse transaction patterns

- create segmentation systems

- monitor segment involvement and migration

- identify the drivers of customer and/or product profitability

— understand the spatial dimensions of a business

- understand the role of channels

- understand sources of business.

\section{MODELLING}

In order to manage customer communications more effectively it is essential that an organisation can predict 
the behaviour of customers. This modelling capability, as with analytics, needs to be embedded into the campaign management process if intelligence is to drive marketing activities. This modelling capability is concerned with allowing an organisation to:

- predict product or service purchase propensity for up-selling and cross-selling activities

- predict value of product purchase (use to create gamma scores that factor value into product purchase propensity scores)

- predict response time bands (used to manage outward bound calls)

- predict customer and/or product profitability (actual to date, planned and potential)

- predict enquiry potential (used for filtering opportunities)

- predict product or services cancellation

- predict product or service usage dormancy or inactivity

- predict channel preference

- predict customer response to communication activities (single or multi-stage, single or multiple channel).

The modelling environment should support the development, validation, deployment and re-calibration of these models. Fully automating the modelling development process is still fraught with problems so it is not generally used.

\section{COMMUNICATION MANAGEMENT}

Central to the success of any customer relationship strategy is proactive communications with customers. The communication management component of analytical CRM is concerned with the overall management of communications with customers. Traditionally, this type of technology has focused on proactive marketing communications (campaign management), but more and more it is being used to manage other customer communication activities (eg mortgage arrears collection). This is because these technologies can bring structured control and monitoring processes to all customer communications.

This communications management capability is concerned with allowing an organisation to:

- maintain communication reference data, including target key performance indicators (KPIs)

— identify target audiences for communication activity

- prioritise communications across campaigns

- establish control mechanisms such as test and control groups

— initiate communication through the appropriate channel

- maintain communication contact history

- determine direct or inferred responses to communication activities

- monitor campaign and/or communication performance

- automate the communication activities.

This communication management technology is used to support a wide range of communication activities including:

— strategic

- tactical

- customer life stage

- product lifecycle

- trigger event

- statutory

- operational.

The integration of the communication management solution with the other 
communication channels has led to an explosion in integration technologies, which allow real-time, or near real-time, initiation and management of customer communications or dialogue (dialogue is the term used to describe interactive communications, often real or near real time).

Technologies that search out trigger events using sophisticated analytical techniques have emerged. These are sometimes called trigger agents.

\section{PERSONALISATION}

There is an expectation by customers that communications should be personalised. Gone are the days of starting a letter with 'Dear Valued Customer'. This means that communications need to exploit the information that an organisation has about the individual customer (corporate memory) to ensure that communications are timely and relevant. The ability to personalise communications and to respond appropriately to inward bound communications from a customer are essential if companies are to gain their respect. This personalisation capability is concerned with allowing an organisation to:

- select the most appropriate medium for the message and the customer

- use the correct copy style for the customer or segment

— include core (static) or derived (dynamic) data from the corporate repository in the communication

- facilitate call to action

- measure response through effective close loop mechanisms across all the response channels.

Typical features of this type of personalisation technology include:
- personalisation templates

- personalisation rules.

Generally this type of personalisation has been concentrated in the electronic channels, but advances in printing and content management technologies are allowing personalisation techniques to be applied to all communication media including print and voice.

\section{INTERACTION MANAGEMENT}

In the past the focus of attention has been on the management of proactive communications management (outward-bound marketing communications). This is changing as organisations start to recognise the importance of effective management of response to proactive communications or, even more importantly, customer-initiated communications. There is a growing desire, particularly with the real-time electronic channels, to manage this dialogue more effectively. This interaction management capability is concerned with allowing an organisation to:

— add intelligence to the management of inward-bound customer communications

- standardise and personalise the dialogue, especially when it is electronic

- ensure that any dialogue takes into account other interactions (especially recent ones) that the individual has had with the organisation

- monitor the effectiveness of the dialogue

- support the sales process, through visual prompts

- align the nature and tone of the dialogue to the customer or segment profile

- maintain details of any dialogue and outcome. 


\section{OPTIMISATION}

With the wider availability of channels that can be used to communicate with and receive communication from customers, has come a significant increase in the complexity of the communication decisioning process, ie deciding which customer gets what communication, when and by what channel. This has led to the development of a new range of optimisation technologies specifically for marketing. This optimisation capability is concerned with allowing an organisation to:

- define global contact rules that manage the number of communications that a customer will receive within a defined period and the time period between communications of a similar type

- determine the optimal product offering to be made to the customer at a point in time. This opimisation process is typically being driven by behavioural models

- determine the most appropriate channel for the customer communication, taking into account customer preferences and channel and/or business constraints, eg budget

- ensure that the 'best' opportunities are sent to the channel, thereby maximising the productivity of the channel

- allocate opportunities to individual agents within a given channel

- distribute opportunities to primary and secondary channel assignments based on daily channel capacity and number of communication opportunities.

It is this last element of the analytical CRM solution that the rest of this paper explores in more detail.

\section{APPROACH TO OPTIMISATION}

The key thing that the optimisation technologies are trying to do is to mathematically maximise the profit generated from marketing and other communications, removing the guesswork from the campaign management process.

The problem is that with dozens of potential offers, many communication channels and thousands or millions of potential customers, there could be billions of different offer-customerchannel combinations to choose from.

So how can the combination that mathematically maximises profit be found?

Optimisation technologies use mathematical algorithms to determine the best combinations of customers, products, and communication channels to maximise profit, while taking into account real-world business constraints such as limited budget or channel capacity. The output of the optimisation process can then be used to drive the communications with a customer. The optimisation process therefore becomes a crucial step in the communication decisioning process. It allows marketing communication activities to be tuned for maximum financial payback (ROI) across all campaigns. Figure 1 shows how optimisation technologies fit within the marketing communication process.

Data warehousing solutions allow companies to store, organise and retrieve valuable customer data. Analytical and modelling tools can be used to understand the customer and build behavioural models (single dimensional models). For a given marketing offer, these models can be used to determine a customer's likelihood of responding to that offer. But these modelling techniques do not take into account the impact of other concurrent offers, channel or business constraints. This is 


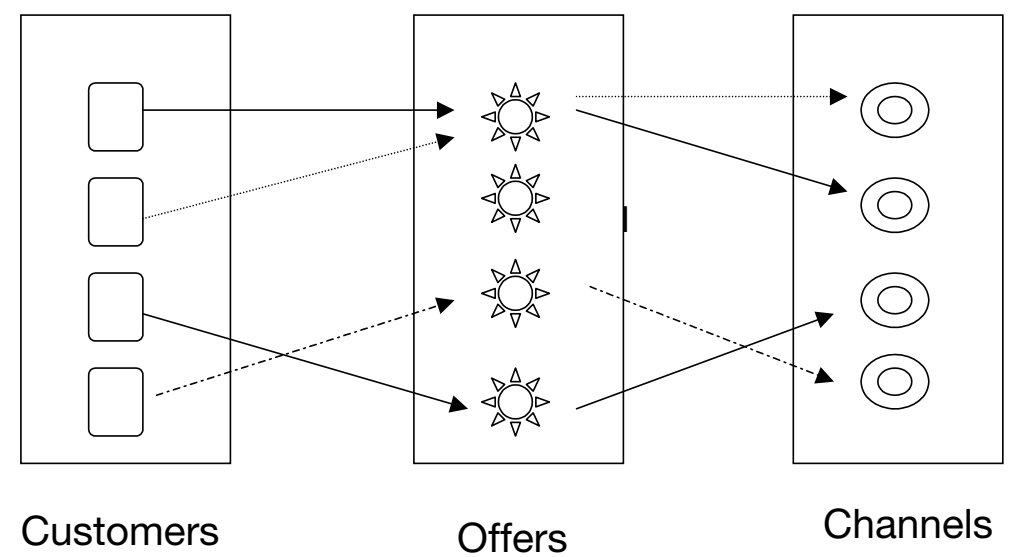

Figure 2 Customer, offer, channel combinations

where optimisation techniques can help to resolve the problem of maximising return on investment (MROI) across all planned communications.

The optimisation technologies can maximise an organisation's overall ROI, or any other specifically defined business objective, by using sophisticated optimisation techniques to do what previously has been impossibly complex: matching the optimal product among many with the optimal customer among thousands, or even millions.

The better solutions take into account interdependencies of financial goals, business constraints and customers' needs to deliver the offer that is best for the business and satisfies the customers.

The optimisation technologies allow the user to define the business constraints, input customer response data and specify which offers and communication channels to consider. The software automatically selects the appropriate combination based on specified objectives, such as maximising profit, then rolls up the projected results and scores the database for offer distribution via the correct channel.

For the first time these optimisation technologies allow an organisation to optimise the value of their integrated marketing campaigns to achieve the maximum financial return. Key features of optimisation technologies include:

- user and pre-defined mathematically definable goals

— interactive expectations analysis

- user-defined constraints using an underlying menu of metrics and variables to determine the most profitable campaigns before execution

- multi-level optimisation at either customer or segment level

- incorporating behavioural changes or triggers into the decisioning process

- integration with existing modelling and communication management technologies

— rapid deployment.

The linear programming optimisation techniques used to manage the supply chain in manufacturing industries, airline yield management, and financial investment risk assessment can only handle relatively small-scale problems and do not work for marketing optimisation.

Business rules and other 'best guess' solutions that were invented to try to address this problem do not mathematically maximise the profit. These techniques do not consider the 
complicated interdependencies of multiple offers, are not easily updated with new information, and cannot scale to the magnitude of the problem. A new class of non-linear optimisation techniques is being developed to meet this current business problem.

It is clear that these new optimisation technologies are adding a new intelligent dimension to the process of managing customer communications. They are likely to add value to the analytical CRM process and will therefore stay. The key vendors to watch in this area are

- Marketswitch,

- NCR and

- SAS.

\section{CONCLUSION}

Customer relationship optimisation $(\mathrm{CRO})$ as a phrase is starting to be seen more and more in the industry. Its use is gaining popularity, particularly among the CRM software vendors, as they try to differentiate their technology in an increasingly homogeneous market. Its adoption by the technology market analysts such as Gartner is giving CRO further credence. The phrase itself causes confusion by not clearly defining what the technology is meant to do.

Below the froth of this marketing hype there are a few companies developing new technologies aimed at optimising some aspect of the marketing communication process. These solutions are still embryonic and are evolving as the nature of the business problem becomes better understood. It is these technologies that warrant close monitoring and, in the cases above, serious investigation.

CShaun Doyle 2001

\section{Acknowledgments}

NCR, 1700 S. Patterson Blvd., Dayton, Ohio 45479, USA; website: ncr.com.

Gartner, 56 Top Gallant Road, PO Box 10212, Stamford, CT 06904, USA; tel: +1 203.964.0096; website: gartner.com.

MarketSwitch Corporation, 108 Powers Court, Dulles, VA 20166, USA; tel: +1 (703) 444-6750; website: marketswitch.com.

SAS Institute Inc., SAS Campus Drive, Cary, NC 27513-2414 USA; tel: +1 (919) 677 8000; website: sas.com. 